\title{
BASIS GRAPHS OF PREGEOMETRIES
}

\author{
BY STEPHEN B. MAURER \\ Communicated by Gian-Carlo Rota, December 18, 1972
}

A combinatorial pregeometry, or matroid, may be defined as a finite set of elements $E$ and a collection of bases $\mathscr{B}$, all subsets of $E$, such that for all $B, B^{\prime} \in \mathscr{B}$ and any $e^{\prime} \in B^{\prime}-B$, there exists $e \in B-B^{\prime}$ for which $B-e+e^{\prime} \in \mathscr{B}$. This exchange axiom suggests it is fruitful to represent a pregeometry $\mathscr{M}$ by a graph: Let there be a vertex for each basis and an edge for each pair of bases differing by a single exchange. We get the basis graph $B G(\mathscr{M})$. A special case of this construct, tree graphs, has been studied for several years [3]. The more general situation has attracted attention only recently $[1],[4]$.

Our purpose here is to announce our own studies of pregeometry basis graphs [6], [7], and to state some of our key findings. We have recently learned that some of our results and methods are similar to those discovered about the same time by Cunningham [2] and also by Holzmann, Norton and Tobey [5]. In particular, Theorems 2 and 3 below are in this category.

We first characterize basis graphs. Given any graph $G(\mathscr{V}, \mathscr{E})$, suppose $\delta\left(v^{\prime}, v^{\prime \prime}\right)=2$ and $\mathscr{V}^{\prime} \subset \mathscr{V}$ consists of $v^{\prime}, v^{\prime \prime}$ and all vertices adjacent to both. Then the induced subgraph $\left\langle\mathscr{V}^{\prime}\right\rangle$ is called the common neighbor subgraph $C N\left(v^{\prime}, v^{\prime \prime}\right)$, or simply a $C N$. In a basis graph each $C N$ is either a square (4-cycle), a pyramid (with square base), or an octahedron. Again in any graph, a leveling from $v_{0}$ is a partition of $\mathscr{V}$ into

$$
\mathscr{V}_{k}=\left\{v: \delta\left(v, v_{0}\right)=k\right\}, \quad k=0,1, \ldots
$$

In any leveling of a basis graph, each octahedral $C N$ lies in one of three positions : (i) all in one level; (ii) across two levels, three adjacent vertices in each; or (iii) across three levels, one vertex in the highest, one not adjacent to it in the lowest, and four in between. Any other $C N$ must lie as would an induced subgraph of such an octahedron. We call this the positioning condition. Finally, the neighborhood subgraph $N(v)$ is the induced subgraph on the vertices adjacent to $v$ ( $v$ not included).

THEOREM 1. G is a basis graph iff

(1) it is connected;

(2) each $C N$ is a square, pyramid or octahedron;

AMS (MOS) subject classifications (1970). Primary 05B35; Secondary 05C05, 05C35, 05B20, $15 \mathrm{~A} 30$.

Key words and phrases. Pregeometry, matroid, basis graph, tree graph, combivalence class, pivoting. 
(3) in every leveling, every $C N$ meets the positioning condition; and

(4) for some $v_{0}, N\left(v_{0}\right)$ is the line graph of a bipartite graph.

There is an alternate form of this characterization in which the positioning condition is assumed for the leveling from $v_{0}$ only, but another condition forbidding two particular induced subgraphs is added. We can also show that (4) is redundant in many cases. We suspect it is redundant in all. Indeed, we conjecture that (1) and (2) alone suffice if there are no square $C N$ 's; unfortunately, they do not suffice otherwise.

The proof of Theorem 1 leads quickly to two other results. First, there is a simple characterization of basis graphs in terms of mappings into the small subclass whose $C N$ 's are all octahedra. Second, if two paths differing by a single vertex are considered homotopic, all basis graphs are homotopically trivial. More generally, this homotopy relates to graph products in the same way ordinary homotopy relates to topological products, i.e.,

$$
\pi\left(G_{1} \times G_{2}\right) \approx \pi\left(G_{1}\right) \times \pi\left(G_{2}\right) .
$$

We characterize pregeometries whose basis graphs contain only one or two types of $C N$ 's. The most interesting of these results is that $\mathscr{M}$ is binary (see [9] or [11]) iff $B G(\mathscr{M})$ contains no induced octahedra.

Given $G(\mathscr{V}, \mathscr{E}),\left\langle\mathscr{V}^{\prime}\right\rangle$ is shortest path complete (SPC) if whenever $v \in \mathscr{V}$ is on a shortest path in $G$ between $v^{\prime}, v^{\prime \prime} \in \mathscr{V}^{\prime}$, then $v \in \mathscr{V}^{\prime}$. Tutte [9] has characterized certain important classes of pregeometries in terms of forbidden minors. We show that minors of $\mathscr{M}$ correspond to SPC's of $B G(\mathscr{M})$. This allows us to find some analogues of his theorems. For instance, $\mathscr{M}$ is regular iff no SPC of $B G(\mathscr{M})$ is an octahedron or a certain graph with 29 vertices. Planar-graph pregeometries can be characterized by further requiring that no SPC be the tree graph of $K_{5}$ or $K_{3,3}$. However, graphic pregeometries cannot be characterized in this way, for a pregeometry and its dual have isomorphic basis graphs.

For any $\mathscr{M}(E, \mathscr{B})$ we may assign to each $B \in \mathscr{B}$ a $0-1$ circuit matrix $C(B)$ with rows indexed by $B$, columns by $D=E-B$, and a 1 in entry $(b, d)$ iff $B-b+d \in \mathscr{B}$. For graphic pregeometries these are closely related to the usual cycle and cocycle matrices. More generally, for each binary pregeometry, one can get from any circuit matrix to any other by the standard pivoting rules of linear programming (applied to the field $F_{2}$ ). In fact, $\{C(B): B \in \mathscr{B}\}$ is just a combivalence class as defined by Tucker [8]. For arbitrary pregeometries, one may still pivot between circuit matrices; with just one exception the rules are the same as for a combivalence class. We use this result, first demonstrated by Yoseloff [10], to obtain simply several propositions. Among these are the next theorem and the results already mentioned on basis graphs with restricted $C N$ 's. 
$\mathscr{M}(E, \mathscr{B})$ is proper if no element is in every basis or outside of every one. Improper elements do not affect $B G(\mathscr{M})$. Also, if there exist $\mathscr{M}_{i}\left(E_{i}, \mathscr{B}_{i}\right)$, $i=1,2$, where the $E_{i}$ are nonempty and partition $E$, and $B=\left\{B_{1} \cup B_{2}\right.$ : $\left.B_{i} \in \mathscr{B}_{i}\right\}$, then $\mathscr{M}$ is a nontrivial sum.

THEOREM 2. If $\mathscr{M}$ is proper, the following are equivalent:

(1) $\mathscr{M}$ is a nontrivial sum;

(2) $B G(\mathscr{M})$ is a nontrivial product;

(3) for some $B, N(B)$ in $B G(\mathscr{M})$ is disconnected.

We also use circuit matrices to study polars, the tops and bottoms of levelings. Not only is every polar itself a basis graph, it is even the basis graph of a sum. This is trivially true for a top polar, since it is a single vertex. However, we generalize the notion of leveling in a way which untrivializes this fact, while at the same time bringing out an up-down symmetry.

Finally, we have noted that $\mathscr{M}$ and its dual $\mathscr{M}^{*}$ have isomorphic basis graphs. There is a partial converse. We write $\mathscr{M} \approx \mathscr{M}^{\prime}$ if there is a bijection of elements which preserves bases.

THEOREM 3. If $\mathscr{M}, \mathscr{M}^{\prime}$ are proper and $B G(\mathscr{M}) \approx B G\left(\mathscr{M}^{\prime}\right)$, then there exist $\mathscr{M}_{1}, \mathscr{M}_{2}$ where

$$
\mathscr{M}=\mathscr{M}_{1}+\mathscr{M}_{2}, \quad \mathscr{M}^{\prime} \approx \mathscr{M}_{1}+\mathscr{M}_{2}^{*} .
$$

REMARK. In many situations it is natural to restrict attention from pregeometries to geometries; see Crapo and Rota [11]. However, this is usually not the case when one works with bases, where the restriction is that each pair of elements must appear in some basis. In particular, there does not seem to be a nice refinement of Theorem 1 which characterizes basis graphs of geometries.

ACKNowledgement. I wish to thank Professor A. W. Tucker, my thesis adviser at Princeton, for his encouragement and suggestions.

\section{REFERENCES}

1. J. A. Bondy, Pancyclic graphs. II, University of Waterloo, (summary in Proc. Second Louisiana Conf. Combinatorics, Graph Theory and Computing, Baton Rouge, La., 1971).

2. W. Cunningham, The basis graph of a matroid, University of Waterloo, (unpublished).

3. C. A. Holzmann, Survey on tree graphs, Proc. IEEE International Symp. Circuit Theory, Los Angeles, April 1972 (to appear).

4. C. A. Holzmann and F. Harary, On the tree graph of a matroid, SIAM J. Appl. Math. 22 (1972), 187-193.

5. C. A. Holzmann, P. G. Norton and M. D. Tobey, A graphical representation of matroids, SIAM J. Appl. Math. (to appear).

6. S. B. Maurer, Matroid basis graphs, Ph.D. Thesis, Princeton University, May 1972. (Available as a Math. Dept. Technical Report.)

7. —, Matroid basis graphs. I, II, J. Combinatorial Theory, Ser. B. (to appear).

8. A. W. Tucker, A combinatorial equivalence of matrices, R. Bellman and M. Hall, (editors) Combinatorial Analysis, Proc. Symp. Appl. Math. 10, Amer. Math. Soc., 
Providence, R.I., 1960, pp. 129-140. MR 22 \#5579.

9. W. T. Tutte, Lectures on matroids, J. Res. Nat. Bur. Standards 69B (1965), 1-47. MR 31 \# 4023.

10. M. L. Yoseloff, Matroids as pivotal algebraic systems, Math. Dept. Technical Report, Princeton University, 1970.

11. H. H. Crapo and G.-C. Rota, On the foundations of combinatorial theory: Combinatorial geometries, MIT Press, Cambridge, Mass., 1970.

Department of Mathematics, Princeton University, Princeton, New Jersey 08540 03833

Department of Mathematics, Phillips Exeter Academy, Exeter, New Hampshire

Current address: Department of Combinatorics and Optimization, University of Waterloo, Waterloo, Ontario, Canada N2L 3GI (effective July 1) 